\title{
Increased incidence of neurological complications in patients receiving an allogenic bone marrow transplantation from alternative donors
}

\author{
C de Brabander, J Cornelissen, P A E Sillevis Smitt, Ch J Vecht, M J van den Bent
}

\begin{abstract}
Objective-To compare the frequency and type of neurological complications after bone marrow transplantation (BMT) with an HLA identical unrelated donor or a mismatched related donor (alternative donors) to the neurological complications after matched sibling BMT for standard and high risk leukaemia or myelodysplastic syndromes.
\end{abstract}

Methods-Retrospective analysis of consecutively treated patients with $(a)$ BMT from alternative donors $(n=39), \quad(b)$ treated with matched sibling BMT for standard risk leukaemia, myelodysplastic syndromes, or aplastic anaemia $(n=53)$, and $(c)$ treated with matched sibling BMT for high risk leukaemia, myelodysplastic syndromes, or aplastic anaemia $(n=49)$.

Results-A total of 72 neurological complications were found. Most of these occurred within the first 6 months after transplant. Thirty six patients developed a severe neurological complication: 17 Alternative donor patients $(44 \%)$ by contrast with six standard risk patients $(11 \%)$ and 13 high risk patients $(27 \% ; p<0.005)$. The most frequent complication was a metabolic encephalopathy occurring in $18 \%$ of patients. Most of the encephalopathies were caused by either the transplant procedure, cyclosporin, systemic infections, microangiopathic thrombopathy, or by complications induced by graft versus host disease. Infections of the CNS developed in $9 \%$ of patients, cerebrovascular lesions in $3 \%$.

Neuro-Ont of Dr Daniel den Hoed Cancer Center, Rotterdam, the Netherlands $C$ de Brabander P A E Sillevis Smitt Ch J Vecht $\mathrm{MJ}$ van den Bent

Department of Hematology J Cornelissen

Correspondence to: Dr M J van den Bent, Department of Neuro-Oncology, Dr Daniel den Hoed Cancer Center, PO Box 5201, 3008 AE Rotterdam, the Netherlands email: bent@neuh.azr.nl

Received 23 December 1998 and in final form

5 August 1999

Accepted 20 August 1999

-Severe neurological complications are more frequent after BMT from alternative donors. This is mainly due to increased treatment related morbidity and to more profound immunosuppression after BMT from alternative donors.

(F Neurol Neurosurg Psychiatry 2000;68:36-40)

Keywords: bone marrow transplantation; neurological complications; alternative donors

High dose chemotherapy with total body irradiation followed by either autologous or allogenic bone marrow transplantation (BMT) is an effective treatment for various haematological disorders. Unfortunately, an HLA identical sibling donor is only available for a few patients due to matching criteria. Some of these patients can be treated with allogenic
BMT from alternative donors: HLA mismatched sibling donors and HLA-identical unrelated donors. However, allogeneic BMT from alternative donors is associated with considerable morbidity and treatment related mortality. Risk factors for treatment related complications and mortality include older age, type, and risk status of underlying disease, degree of HLA matching, donor type, and comorbidity factors of the patient. Major complications which contribute to treatment related mortality are opportunistic infections, graft versus host disease, interstitial pneumonitis, and veno-occlusive disease..$^{1-3}$

Neurological complications are an important cause of treatment related morbidity and may contribute to it. The few studies that considered these complications are difficult to compare due to differences in patient selection, criteria for neurological complications, or type of BMT. Two often quoted reports on neurological complications of BMT are postmortem studies. ${ }^{45}$ Another study only considered neurological complications of BMT in childhood. ${ }^{6}$ Studies on consecutively treated adults are rare. ${ }^{7-9}$ These studies suggest that the incidence and type of neurological complications are related to the type of transplantation (allogenous $v$ autologous) and to the type of disease. No study has specifically investigated frequency and type of these complications in BMT from alternative donors. We investigated retrospectively the incidence and outcome of neurological complications in these patients, and compared these to the neurological complications occuring in cohorts of standard and high risk BMT patients treated with an allograft from HLA identical sibling donors.

\section{Methods}

The records of all patients who received an allogenic BMT from an alternative donor or treated with a BMT from an HLA identical sibling for high risk leukaemia or myelodysplastic syndromes at the Daniel den Hoed Cancer Center, Rotterdam were reviewed. A group of 50 consecutively treated patients with standard risk aplastic anaemia treated in the same period served as a control group. The patients were classified into these three categories according to donor type and risk status of underlying disease:

(1) Alternative donors-Patients who received an allogenic graft from either an HLA matched unrelated donor or an HLA mismatched related donor. risk leukaemia and all patients with standard 
Table 1 Patient and treatment characteristics of the three treatment groups

\begin{tabular}{lccc}
\hline & Alternative donor & High risk & Standard risk \\
\hline No of patients & 39 & 49 & 53 \\
Mean age (range) & $31(14-48)$ & $41(15-56)$ & $36(16-56)$ \\
Sex: & 23 & 27 & 32 \\
$\quad$ Male & 16 & 20 & 20 \\
$\quad$ Female & 8 & & \\
Primary diagnosis: & 10 & 6 & 17 \\
ALL & 7 & 17 & 20 \\
AML & 9 & 14 & 13 \\
MDS/MPS & 5 & 2 & 3 \\
CML & 37 & 37 & 50 \\
Aplastic anaemia & 3 & 10 & 6 \\
GVHD Prophylaxis & & 2 & 1 \\
T cell depletion + CyA & & 1 & \\
MTX + CyA & 4 & 8 & 35 \\
T cell depletion & 22 & 21 & 17 \\
CyA & 10 & 12 & 0 \\
Conditioning: & 3 & 8 & 1 \\
Cyclo-TBI & 31 & 0 & 0 \\
Cyclo-TBI + Ara-C & & & \\
Cyclo-TBI + VP-16 & & & \\
Cyclo, busulphan & ATG & &
\end{tabular}

$\mathrm{ALL}=$ acute lymphoblastic leukaemia; $\mathrm{AML}=$ acute myeloid leukaemia; $\mathrm{CML}=$ chronic myeloid leukaemia; $\mathrm{MDS} / \mathrm{MPS}=$ myelodysplastic - /myeloproliferative syndrome; $\mathrm{BM}=$ bone marrow; GVHD: graft versus host disease; MTX=methotrexate; CyA=cyclosporine A; Cyclo=cyclophosphamide; $\mathrm{ATG}=$ conditioning regimen also included antithymocyte globulins; TBI=total body irradiation

Table 2 Neurological complications found in the three treatment groups

\begin{tabular}{lcccc}
\hline Complication & $\begin{array}{l}\text { Alternative } \\
\text { donor }(n=39)\end{array}$ & $\begin{array}{l}\text { High risk } \\
(n=49)\end{array}$ & $\begin{array}{l}\text { Standard } \\
\text { risk }(n=53)\end{array}$ & $\begin{array}{l}\text { Total } \\
(n=141)\end{array}$ \\
\hline CNS infection & 6 & 4 & 2 & 12 \\
Encephalopathy & $12^{\star}$ & 11 & 5 & 28 \\
Vascular complications & 3 & 1 & 0 & 4 \\
Other & 8 & 7 & 13 & 28 \\
Total & 29 & 23 & 20 & 72 \\
\hline
\end{tabular}

^Two patients had two episodes with an encephalopathy.

(2) Standard risk patients - patients who received an allogenic graft from an HLA identical sibling donor in first chronic phase chronic myeloid leukaemia, or for untreated aplastic anaemia.

(3) All other patients with leukaemia (with more advanced disease status), patients with myelodysplastic syndromes, and patients with previously treated aplastic anaemia receiving a BMT from an HLA identical sibling donor were considered high risk patients.

The medical records of all patients were reviewed and the data evaluated were: age, sex, primary disease, conditioning regimen, graft versus host disease prophylaxis, and the occurrence, nature, and outcome of neurological complications. The registration of neurological complications began with the start of the conditioning regimen; for this study complications occurring at the time of disease relapse or thereafter were not considered. The complications were divided into four categories: infections of the CNS, encephalopathic disorders (including drug induced seizures), cerebrovascular complications and "other" complications. By definition, (a) all syndromes with decreased level of consciousness due to sys- temic organ dysfunction without structural abnormalities on CT or MRI, $(b)$ drug induced seizures, and (c) posterior leukoencephalopathy syndromes ${ }^{10}$ were considered encephalopathies. Less severe drug induced CNS complications without decreased levels of consciousness such as extrapyramidal syndromes were reckoned among the "other" complications. For this study, cutaneous Herpes zoster infections were not considered. All cases of cyclosporin induced tremor are mentioned separately. Complications were graded according to the NCIC common toxicity criteria (CTC), revised version (1994). Grade 3 (severe) and grade 4 (life threatening) complications were considered as "serious" complications. To allow for sufficient follow up and documentation of neurological complications the interval between the time of transplant and this survey was at least 1 year. For comparison between groups a two tailed $\chi^{2}$ test with Yates' correction was used, $\mathrm{p}<0.05$ was considered significant.

\section{Results}

Between 1983 and 1995, 39 patients received an allogeneic bone marrow graft from an alternative donor, 49 BMT patients were considered high risk patients; the standard risk group consisted of 53 patients. Patient characteristics are summarised in table 1 . At the time of evaluation, 14 alternative donor patients (36\%), 20 high risk patients (41\%), and 28 standard risk patients (53\%) were alive. Table 2 summarises all neurological complications of the different groups. Fifty nine patients (42\%) had a total of 72 neurological complications, most of which occurred within the first 6 months after the transplantation procedure. Table 3 shows the number of serious complications. Seventeen alternative donor patients (44\%) compared with six standard risk patients $(11 \%)$ and 13 high risk patients (27\%; $\mathrm{p}<0.005)$ had a neurological complication that met the criteria for severe or life threatening. Four alternative donors and two high risk patients developed two serious complications. In univariate analysis, no relation was found between the occurrence of serious complications and sex $(\mathrm{p}=0.93)$ or age $(\leqslant 40$ years $v>40$ years; $p=0.98)$. Logistic regression analysis showed no relation between diagnosis and the occurrence of serious complications $(p=0.19)$, correction for BMT group did not alter this conclusion.

Twenty six patients (18\%) developed a metabolic encephalopathy: 10 alternative donor patients $(26 \%), 11$ high risk patients $(21 \%)$, and five standard risk patients $(9 \%)$. In one third of cases the encephalopathy was part of a terminal event. After exclusion of terminal

Table 3 Number of serious complications per patient in the three treatment groups

\begin{tabular}{lllll}
\hline & $\begin{array}{l}\text { One serious } \\
\text { complication }\end{array}$ & $\begin{array}{l}\text { Two serious } \\
\text { complications }\end{array}$ & $\begin{array}{l}\text { Total No of serious } \\
\text { complications }\end{array}$ & Total No of patients \\
\hline Alternative donor $(\mathrm{n}=39)$ & 13 & 4 & 21 & $17(\mathrm{p}=0.0010)^{\star}$ \\
High risk leukaemia $(\mathrm{n}=49)$ & 11 & 2 & 15 & $13(\mathrm{p}=0.089)^{\star}$ \\
Standard risk leukaemia $(\mathrm{n}=53)$ & 6 & 0 & 6 & 6 \\
\hline
\end{tabular}

${ }^{*} \mathrm{p}$ Values of the comparison with the standard risk group. 
encephalopathies, seven alternative donor patients, seven high risk patients, and two standard risk patients developed an encephalopathy. Three alternative donor patients and one high risk patient developed severe cyclosporin encephalopathies, with visual disturbances, hypertension, and seizures. Two of them had cyclosporin concentrations within the therapeutic range, all recovered after stopping CyA, or dose reduction. One patient of the alternative donor group and three high risk patients developed a metabolic encephalopathy as a direct result of the BMT conditioning regimen. Two of these patients (both with high risk leukaemia) developed seizures after the administration of busulfan, despite prophylactic antiepileptic treatment. ${ }^{11}$ The encephalopathy was related to systemic infections in seven patients, in seven to multiorgan dysfunction or failure, and in three to severe systemic graft versus host disease. In three patients it was caused by a thrombotic microangiopathy, which proved fatal in two. The other patient developed a posterior leukoencephalopathy syndrome with cortical blindness which was reversible on plasmapheresis.

Twelve patients developed a CNS infection, six in the alternative donor group, four in the high risk group, and two in the standard risk group. Three cases of a cerebral toxoplasmosis infection were seen, all in alternative donor patients. One of these patients died of a rapidly progressive systemic infection, the other two recovered after treatment. Three patients developed a bacterial meningitis, one of whom died. They were caused by Pseudomonas aeroginosa, Hemophilus influenzae, and Pneumococcus respectively, and occurred on day 116 , day 180 , and day 317. Two patients with multiple intracerebral abscesses died from other complications (respiratory failure, severe graft versus host disease) before a causative agent could be identified; necropsy was refused in both cases. One case of EBV encephalitis with full recovery after treatment with ganciclovir has been previously reported. ${ }^{12}$ No causative micro-organism could be established in three patients with probable viral meningoencephalitis, both were treated with broad spectrum antibiotics and acyclovir. The outcome was fatal in one but the others made a full recovery. Three alternative donor patients and one high risk patient developed cerebrovascular complications. One patient had multiple haemorrhagic infarcts during fatal multiple organ failure. One patient had seizures due to a haemorrhage in the right occipital lobe, one patient developed a cortical vein thrombosis, and one patient had transient ischaemic attacks for which no cause was established.

Various less severe complications were noted. Nine patients had less serious but potentially dangerous cyclosporin toxicity, consisting of varying combinations of headache, visual disturbances, an increased blood pressure, and tremor. One patient had a cyclosporin induced extrapyramidal syndrome; he recovered completely after discontinuation. Seven cases of other drug induced extrapyramidal disorders (all caused by antiemetics) were found. All but one recovered with biperiden or a dose reduction of the offending drug. Six patients had mild distal sensory motor neuropathy after BMT. Three patients had Bell's palsy, one patient had a mild peroneal neuropathy, and one patient developed Lhermitte's sign. A cyclosporin related tremor was seen in 16 patients $(11 \%)$.

\section{Discussion}

This study provides a detailed survey of neurological complications after BMT in 39 consecutively treated patients with grafts from alternative donors and of 49 high risk patients treated with a BMT from an HLA identical sibling. These were compared to the neurological complications found in a group of 53 consecutively treated standard risk patients. Although the type of neurological complications did not differ between the three groups of patients, severe complications were more frequent after BMT from alternative donors compared with BMT for standard risk patients $(p<0.005)$. Some caution in the interpretation of the results of this retrospective survey is warranted, as this study is not a randomised or matched comparison. Such a comparison is virtually impossible, due to the many differences between patients for disease and previous treatments.

The increased incidence of neurological complications in alternative donor patients reflects the higher general treatment related morbidity and mortality associated with BMT using alternative donors for BMT for high risk disease. ${ }^{13}$ An important cause for the increased morbidity in alternative donor recipients is the increased susceptibility for opportunistic infections compared with HLA matched sibling BMT. ${ }^{14}{ }^{15}$ The use of antithymocyte globulins in alternative donor BMT for prevention of graft rejection is a major difference in conditioning regimen between the treatment groups, which further impairs immune reconstitution after BMT. Immune reconstitution is also more impaired in patients receiving a graft which is not fully HLA matched. ${ }^{16}$ The incidence of severe neurological complications seems slightly higher in high risk patients. Most of the high risk patients have undergone several lines of chemotherapy before the BMT. This increased intensity of pretreatment may account for the marginal increase of severe neurological complications.

Others found a significant impact of CNS complications on survival. ${ }^{9}$ Apart from the CNS infections the neurological complications did not contribute to the treatment related mortality in our patients. The prognosis of encephalopathies was mainly determined by the underlying cause, if this was reversible a good neurological recovery was the rule. The neurological complications, however, did cause considerable morbidity, even if they were less severe.

As in other series, the most frequent neurological complications in our patients were metabolic encephalopathies. They were found in $9 \%$ of standard risk patients, but in no less than $26 \%$ of patients with alternative donors 
and in $21 \%$ of the high risk patients. In other series on allogenic BMT for both standard and high risk leukaemia, $5 \%-14 \%$ of patients developed encephalopathies and seizures. ${ }^{689} \mathrm{~A}$ study on autologous BMT in Hodgkin's disease reported metabolic encephalopathies in $27 \%$ of patients in the first 6 weeks after BMT, most of which were secondary to opportunistic lung infections and respiratory failure. ${ }^{7}$ However, any comparison with other studies is difficult as the reported incidence of neurological complications is also dependent on the definition of such complications. We only took those neurological complications into account which were subject to further neurological investigation, and disregarded patients with, for example, terminal hepatic failure and encephalopathy in whom treatment had already been discontinued. We also disregarded neurological complications at the time of tumour relapse or thereafter. Other sources for variation of incidence of neurological complications may be attributed to differences in patient selection and management of BMT (for example, the use of $\mathrm{T}$ cell depleted transplants). It is also often difficult to identify the exact cause of encephalopathy after BMT. Many patients have multiple complications at the same time-for example, sepsis with organ dysfunction, and are treated simultaneously with neurotoxic drugs such as cyclosporin.

The causes of metabolic encephalopathy in our patients included systemic infections, treatment related organ failure including graft versus host disease, and side effects of cyclosporin. Cyclosporin A (CyA) plays a central part in prevention and treatment of acute graft versus host disease. It may cause various neurological side effects, such as headache, tremor, seizures, paraparesis, cerebellar symptoms, somnolence, and a parkinsonian syndrome ${ }^{17-22}$ Many of these symptoms are a manifestation of the posterior leukoencephalopathy syndrome, characterised by an increase in blood pressure, headache, visual disturbances (such as cortical blindness), and seizures. Brain CT and MRI usually show white matter changes, predominantly in the occipital lobes. ${ }^{10}$ Neurotoxicity induced by CyA has been associated with hypomagnesaemia, hypercholesterolaemia, hyperlipidaemia, and concurrent high dose steroid therapy. ${ }^{202-25}$ Four of our patients had a full blown posterior leukoencephalopathy syndrome, eight other patients developed only mild signs and symptoms of CyA encephalopathy. One patient had an extrapyramidal syndrome which proved reversible on withdrawal of CyA. A postural tremor is the most frequent neurological complication of CyA, in this group it was noted in $12 \%$ of patients.

Infections of the CNS are the second most important source of neurological complications after BMT. Eight per cent of our patients developed CNS infections: $15 \%$ of all alternative donor patients, $8 \%$ of high risk patients and $4 \%$ of standard risk patients. In series on adult BMT recipients, two studies on autologous BMT did not report CNS infections, and CNS infections were reported in $1 \%-8 \%$ of patients after allogenic BMT. ${ }^{7-9}{ }^{26}$ In this series, all CNS toxoplasmosis occurred in alternative donor patients. Other BMT series reported a $1 \%-2 \%$ CNS toxoplasmosis rate. ${ }^{6827} 28$ The high rate of CNS infections in our alternative donor BMT recipients may be explained by the more profound immunosuppression in these patients after the use of ATG and the more profound $\mathrm{T}$ cell depletion for prevention of graft rejection

Similar to our findings, in most studies cerebrovascular complications (usually haemorrhagic) are present in $2 \%-4 \%$ of patients. ${ }^{82629}$ Cerebral infarctions after BMT are associated with non-bacterial thrombotic endocarditis, but we found no evidence for this in our patient with recurrent transient ischaemic attacks. ${ }^{4} 50$

Three patients in this series developed a Bell's palsy. A peripheral facial nerve dysfunction in patients with a haematological malignancy suggests leptomeningeal metastasis, but all three patients recovered without treatment. Recent investigations have suggested that infections with HSV-1 are the major cause of Bell's palsy. ${ }^{31}$ To the best of our knowledge, Bell's palsy has not been reported in BMT recipients, nor is it more frequent in other immunocompromised conditions.

In conclusion, Alternative donor patients are more prone to develop severe neurological complications than standard risk patients. High risk patients have a neurological complication rate in between those two groups. Encephalopathies and CNS infections account for most of the severe complications. Most likely, the increased incidence of neurological complications in alternative donor patients is due to the more profound and more prolonged immunosuppression resulting in an increased susceptibility for opportunistic infections after alternative donor BMT. Although the neurological complications add to the increased morbidity in alternative donor patients, the prognosis of these complications depends on the underlying cause, in only a few patients the neurological complication itself is the cause of a fatal outcome.

We gratefully acknowledge the statistical assistence of W L J van Putten.

1 O'Reilly RJ, Hansen JA, Kurtzberg J, et al. Hematology 1996. Education Program of the American Society of Hematology. Orlando, Florida: ASH, 1996:132-46.

2 Goulden NJ, Cornish JMM, Potter MN, et al. Bone marrow transplantation from volunteer unrelated donors. Cancer transplantation from volum
Treat Rev 1995;21:325-53.

3 Anasetti C, Etzioni R, Petersdorf EW, et al. Marrow Anasetti C, Etzioni R, Petersdorf EW, et al. Marrow Med 1995;46:444-51.

4 Mohrmann RL, Mah V, Vinters HV. Neuropathologic findings after bone marrow transplantation. Hum Pathol 1990; 21:630-9.

5 Patchell RA, White III CL, Clark AW, et al. Neurologic complications of bone marrow transplantation. Neurology 1985;35:300-6.

6 Wiznitzer M, Packer RJ, August CS, et al. Neurological complications of bone marrow transplantation in childhood. Ann Neurol 1984;16:569-76.

7 Snider S, Bashir R, Bierman P. Neurologic complications after high-dose chemotherapy and autologous bone marrow transplantation for Hodgkin's disease. Neurology 1994; row transp $681-4$.

8 Graus F, Saiz A, Sierra J, et al. Neurologic complications of autologous and allogenic bone marrow transplantation in patients with leukemia. Neurology 1996;46:1004-9. 
9 Antonini G, Ceschin V, Morino S, et al. Early neurologic complications following allogenic bone marrow transplant complications following allogenic bone $\mathrm{m}$.

10 Hinchey J, Chaves C, Appignani B, et al. A reversible posterior leukoencephalopathy syndrome. $N$ Engl $\mathcal{f} \mathrm{Med}$ 1996;334:494-500.

11 Salloum E, Khan KK, Cooper DL. Clorambucil induced seizures. Cancer 1997;79:1009-13.

12 Dellemijn PLI, Brandenbrug A, Niesters HGM, et al. Succesful treatment of Epstein Barr meningo-encephalitis following bone marrow transplantation with ganciclovir. Bone Marrow Transplant 1995;16:311-12.

13 Kernan NA, Bartsch G, Ash RC, et al. Analysis of 462 transplantations from unrelated donors facilitated by the national marrow donor program. N Engl f Med 1993;328: 593-602.

14 Marks DI, Cullis JO, Ward KN, et al. Allogenic bone marrow transplantation for chronic myeloid leukemia using sibling and volunteer unrelated donors. Ann Intern Med 1993;119:207-14

15 Ochs L, Shu XO, Miller J, et al. Late infections after allogenic bone marrow transplantation: comparison of incidence in related and unrelated donor transplant recipi995;86:3979-86.

16 Petersdorf EW, Longton GM, Anasetti C, et al. The significance of HLA-DRB1 matching on clinical outcome after HLA-A B, DR identical unrelated donor marrow transplantation. Blood 1995;86:1606-13

17 Rubin AM, Kang H. Cerebral blindness and encephalopathy with cyclosporin A toxicity. Neurology 1987;37:1072-6.

18 Bird GLA, Meadows J, Goka J, et al. Cyclosporin-associated akinetic mutism and extrapyramidal syndrome after liver transplantation. 7 Neurol Neurosurg Psychiatry 1990;53: 1068-71.

19 Steiger MJ, Farrah T, Rolles K, et al. Cyclosporin associated headache. 7 Neurol Neurosurg Psychiatry 1994;57:1258-9.

20 de Groen PC, Aksamitt AJ, Rakela J, et al. Central nervous system toxicity after liver transplantation. $N$ Engl $f \mathrm{Med}$ 1987;317:861-6.
21 Gharpure VS, Hutchinson RM, Durrant STS. Migraine after bone marrow transplantation. Lancet 1990;335:57.

22 Reece DE, Frei-Lahr DA, Shepherd JD, et al. Neurologic complications in allogenic bone marrow transplant patients receiving cyclosporin. Bone Marrow Transplant 1991;8: 393-401.

23 Thompson CB, Sullivan KM, June CH, et al. Association between cyclosporin neurotoxicity and hypomagnesaemia. Lancet 1984;ii:1116-20.

24 Kalhs P, Brugger S, Schwarzinger I, et al. Microangiopathy following allogenic bone marrow transplantation. Transplantation 1995;60:949-57.

25 Erer B, Lucarelli G, Angelucci E, et al. CsA-associated neruotoxicity and ineffective prophylaxis with clonazepam in patients transplanted for thalassemia major: analysis of risk factors. Bone Marrow Transplant 1996;18:157-62.

26 Teive H, Zetola V, Zanis J, et al. Neurological complications in bone marrow transplantation: an analysis of 458 patients [abstract]. Neurology 1996;46:A350.

27 Derouin F, Devergie A, Auber P, et al. Toxoplasmosis in bone marrow-transplant recipients: report of seven cases and review. Clin Infect Dis 1992;15:267-70.

28 Adams D, Denier C, Bourhis JH, et al. Neurologic complications after bone marrow transplantation (BMT): a 5 year retrospective study [abstract]. $\mathcal{F}$ Neurol 1997; (suppl 3):S21.

29 Pomeranz S, Naparstek E, Askenazi E, et al. Intracranial haematomas following bone marrow transplantation. 7 Neurol 1994;241:252-6.

30 Jerman MR, Fick RB. Non-bacterial thrombotic endocarditis associated with bone marrow transplantation. Chest 1986;90:919-22.

31 Murakami S, Mizobuchi M, Nakashiro Y, et al. Bell's palsy and Herpes simplex virus: identification of viral DNA in endoneurial fluid and muscle. Ann Intern Med 1996; 124:27-30. 